\section{A new health technology: where is the consensus on a clinically worthwhile benefit?}

B Foot ${ }^{1,4}$, R Foy ${ }^{2}, U$ Chakravarthy ${ }^{3}$ and

R Wormald ${ }^{4}$

Abstract

Aim New therapies are often introduced into the NHS prior to full evaluation, leading to inequities in provision.

Uncertainty exists regarding the value of photodynamic therapy in the treatment of neovascular age-related macular

degeneration. We ascertained the availability of this treatment and the information used to inform clinical policy.

Methods A postal survey of all clinical directors/lead consultants in the UK sought data on which (if any) patients were referred or treated with PDT by their unit, the sources of evidence informing clinical policy and the threshold of clinical benefit at which respondents would support the use of PDT. Results 123/152 questionnaires were returned. $42 \%$ of units make some provision for PDT on the NHS, including routine provision by $9 \% .14 .5 \%$ of units offer the option of care in the private sector, whilst $\mathbf{2 6 . 5 \%}$ treated or referred no patients. The threshold at which respondents considered introduction of PDT would be justifiable varied widely.

Respondents cited local literature review, advice from clinicians, guidance from the Royal College and information from the pharmaceutical industry as most influential in determining current policy. However, the National Institute for Clinical Excellence (NICE) and the Cochrane Library were anticipated as playing a greater role in shaping future practice.

Conclusions Substantial variation exists in the availability of PDT. Advocates of PDT may interpret our data as an indication of the NHS failing to provide an effective therapy equitably, whilst others may deduce that patients are receiving an underevaluated treatment in routine clinical practice. The differing thresholds at which clinicians believe treatment would be justified may further exacerbate variations and the priority given to PDT.

Eye (2002) 16, 469-471. doi: 10.1038/

sj.eye. 6700024

Keywords: access; photodynamic therapy; service provision; health technology

The introduction of expensive new health technologies poses major problems for the National Health Service. Evidence of clinical and cost-effectiveness is frequently limited and differences in the availability of resources and beliefs concerning effectiveness can contribute to variations in service provision. Furthermore, once established, it is frequently difficult to reduce the use of interventions subsequently demonstrated to be less effective. ${ }^{1}$

Neovascular age-related macular degeneration (ARMD) causes severe visual loss. A recent randomised controlled trial indicated that repeated treatments with photodynamic therapy (PDT) reduce the relative risk of losing three or more lines of visual acuity to 0.72 and six or more lines to $0.62 .^{2}$ A subgroup analysis suggested benefits were confined to people with 'classic' disease. Despite reservations about the reliability of these findings, ${ }^{3}$ there is now pressure for the NHS to provide this therapy routinely. ${ }^{4}$ If treatment were restricted to eyes with classic choroidal neovascularisation (CNV) with no occult disease, then the cost of provision to the NHS would be approximately $£ 14$ million per annum. However, if patients with predominantly classic membranes are also included for treatment, this figure could be up to six times higher.

We surveyed the current provision of PDT
${ }^{1}$ The Royal College of Ophthalmologists London, UK

2Department of Developmental Sciences University of Edinburgh Scotland, UK

${ }^{3}$ Queen's University and Royal Victoria Hospitals Belfast, Northern Ireland UK

${ }^{4}$ Moorfields Eye Hospital London, UK

Correspondence:

B Foot

E-mail: Barny.Foot@ moorfields.nhs.uk Reproductive and 
in the UK NHS and assessed beliefs about what would constitute a worthwhile level of clinical benefit to justify its provision.

\section{Methods and subjects}

We undertook a postal survey of all clinical directors (or lead consultants) in UK ophthalmic units, as identified by the database of the Royal College of Ophthalmologists during September 2000. We requested information about units' treatment and referral policies regarding the use of PDT in neovascular ARMD and sources of information that might influence local policies.

Respondents also indicated the threshold at which they would support the use of PDT. This was measured in numbers needed to treat (NNT) to prevent the loss of three lines of visual acuity for 2 years for one patient, at a given fixed cost per case of $£ 8000$ per patient treated (this figure was estimated using the TAP study treatment protocol). ${ }^{2}$ The questionnaire was pre-tested and piloted prior to use. Associations were tested for with the $\chi^{2}$-test. A probability of less than $5 \%$ was considered significant.

\section{Results}

Following one reminder, 123/152 questionnaires were returned (response rate $81 \%$ ). There was no evidence of response bias by health region $(P=0.82)$ or by hospital type (teaching vs District General) $(P=0.97)$ Forty-nine $(42 \%)$ units provide PDT on at least a

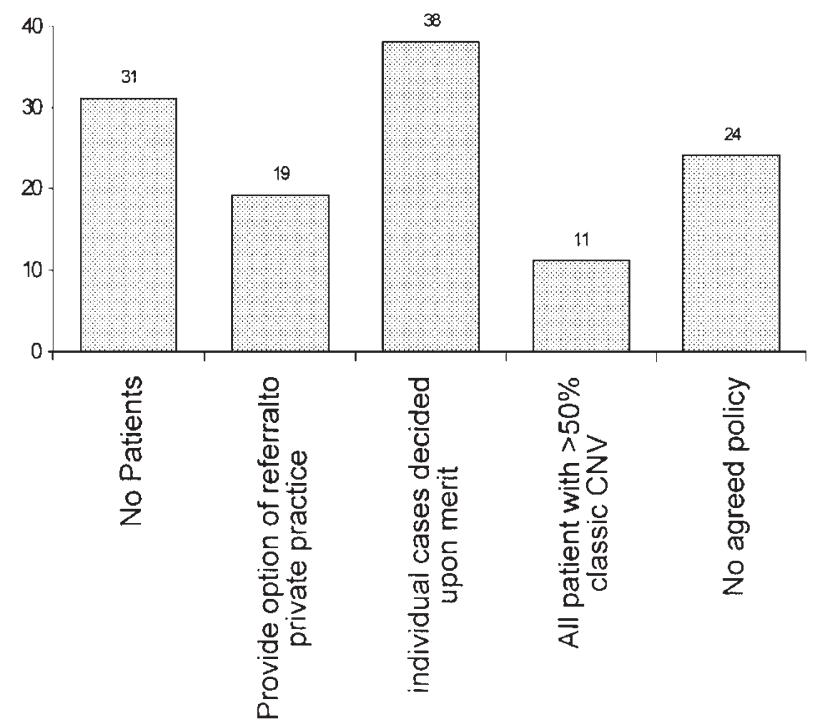

Figure 1 Referral or treatment intentions for PDT in UK hospital units.

limited basis on the NHS, including routine provision by $10(9 \%)$ to patients with majority classic sub-foveal CNV. Nineteen $(14.5 \%)$ units offer patients the option of care in the private sector, whilst 31 (26.5\%) treated or referred no patients (Figure 1).

Local reviews of research literature were reported as informing clinical policy to the greatest degree, ranked within the three most influential sources by 91 respondents, followed by advice from fellow clinicians (84) and Royal College guidance (53) and information from the pharmaceutical industry (44). Cochrane

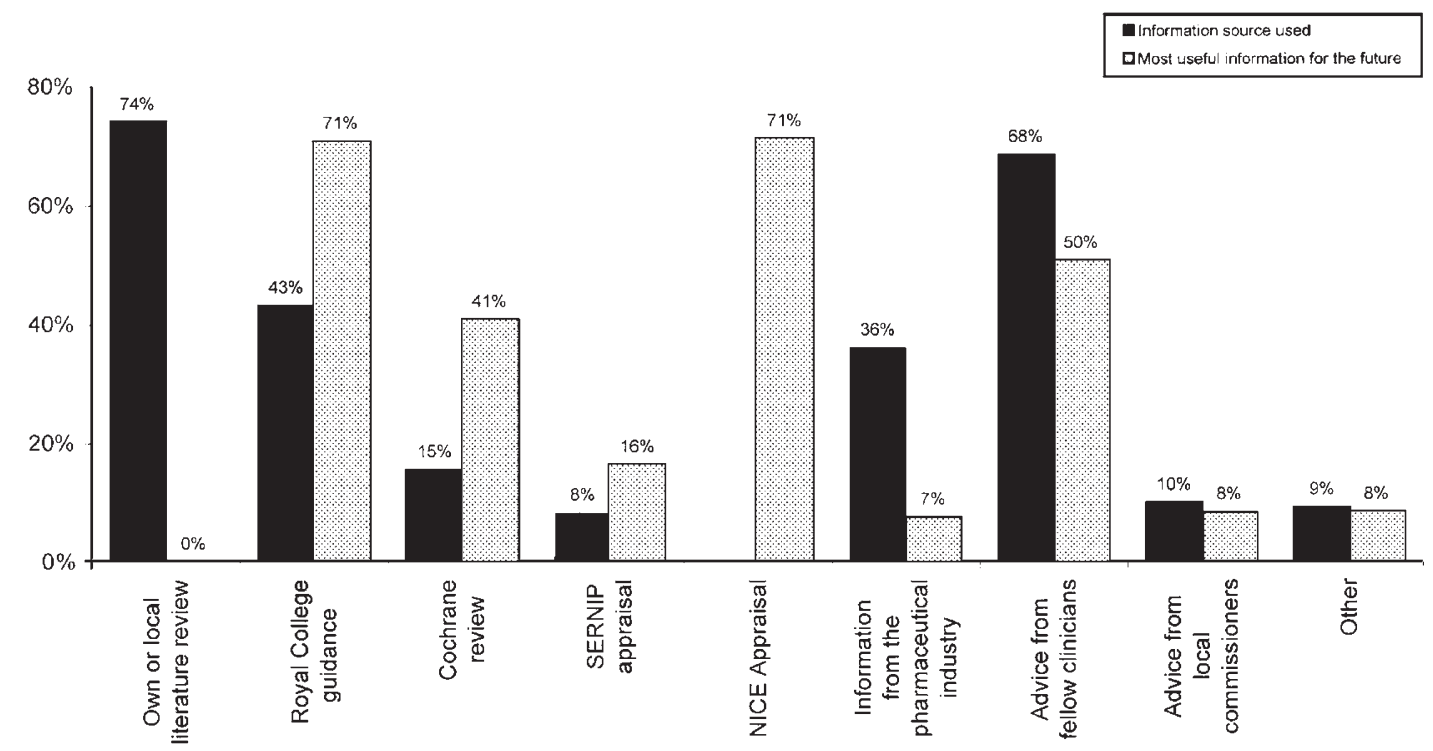

Figure 2 The influence of sources of information (proportion ranked within the top three).

Options for Nice Appraisal and local review not included in both questions. 
Reviews were less commonly ranked among the top three sources (19). There was no significant association between reported service provision and sources of information considered to have been most influential $\left(\chi^{2}=20.5 \mathrm{df}=20, P=0.43\right)$. Information from national sources was generally perceived to have greatest potential for future use. Guidance from the Royal College, NICE, SERNIP and Cochrane systematic reviews were all believed to have a greater forthcoming influence. Information from the pharmaceutical industry and advice from fellow clinicians were perceived to play a lesser role (Figure 2)

At the fixed cost per case, the threshold of benefit at which respondents would support treatment varied considerably (Table 1). One third of respondents would prefer evidence of greater clinical benefit before supporting the use of PDT, whereas $18 \%$ would accept treatment of seven or more patients to prevent the loss of three lines of visual acuity for 2 years for one patient. There was a significant association between the threshold of support and level of service provision $\left(\chi^{2}=20.4, \mathrm{df}=8, P=0.01\right)$.

\section{Discussion}

Clinicians' beliefs about the threshold of effectiveness that would justify the introduction of a new treatment at a given cost varied widely and were associated with inequity of provision and referral policies among departments.

Use of local sources of advice may exacerbate variations in provision and highlights the need for national guidance from a credible source. Clinicians did recognise the value of rigorous reviews of treatment (eg Cochrane Library) and national guidance (National Institute for Clinical Excellence (NICE) and Royal College), but in practice these were either absent or not routinely used. ${ }^{3}$ NICE has reported that an appraisal of PDT will not be available until Summer 2001 at the earliest. However, for any given threshold
Table 1 Threshold of clinical benefit at which treatment with PDT would be supported (in numbers needed to treat)

\begin{tabular}{lc}
$\begin{array}{l}\text { Number of patients requiring } \\
\text { treatment to prevent the loss of } \\
\text { three lines of visual activity for } \\
2 \text { years }\end{array}$ & $\begin{array}{c}\text { Number (\%) supporting use of } \\
\text { PDT at this threshold }(n=116)\end{array}$ \\
\hline & \\
\hline & $22(19)$ \\
7 & $35(30)$ \\
14 & $12(10)$ \\
50 & $6(5)$ \\
100 & $2(2)$ \\
& $1(1)$ \\
Further evidence of & $38(33)$ \\
effectiveness required &
\end{tabular}

of effectiveness, professional opinion concerning what constitutes a worthwhile effect varies and this may augment the present inequity of provision. Furthermore, opinions other than those of ophthalmologists need to be considered. ${ }^{5}$ Future appraisals of new interventions should be preceded by attempts to reach a consensus about what constitutes a clinically important degree of benefit.

\section{References}

1 Mowatt G, Bower DJ, Brebner JA et al. When and how to assess fast-changing technologies: a comparative study of medical applications of four generic technologies. Health Technology Assessment 1997; 1: 1-149.

2 TAP Study Group. Photodynamic therapy of subfoveal choroidal neovascularization in age-related macular degeneration with verteporfin. One year results of 2 randomized clinical trials. TAP report 1. Arch Ophthalmol 1999; 117: 1329-1345.

3 Wormald R, Evans J, Smeeth L. Photodynamic therapy for neovascular age-related macular degeneration (Cochrane review). In: Cochrane Library 1: 2001. Oxford Software Update.

4 Bressler NM. Age-related macular degeneration. New hope for a common problem comes from photodynamic therapy. BMJ 2000; 821: 1425-1427.

5 Steel N. Thresholds for taking antihypertensive drugs in different professional and lay groups: questionnaire survey. BMJ 2000; 320: 1446-1447. 